\title{
Crispr/Cas9 and its current application status on pig breeding
}

\author{
N.H. Tinh ${ }^{1}$, A.P.N. Bui ${ }^{2, *}$, T.T.H. Tran ${ }^{3}$
}

${ }^{1}$ Institute of Animal Sciences for Southern Vietnam, Hiep Thang quarter, Binh Thang ward, Di An town, Binh Duong city; ${ }^{2}$ Faculty of Biotechnology, Ho Chi Minh city Open University, 35 Ho Hao Hon street, Ho Chi Minh city; ${ }^{3}$ Faculty of Applied Sciences, Ton Duc Thang University, 19 Nguyen Huu Tho street, Tan Hung ward, District 7, Ho Chi Minh city, Vietnam

*Corresponding author Tel 0084-8-39300086 Fax: 0084-8-39300085 Email: buiphunamanh@ yahoo.com

Journal of Livestock Science (ISSN online 2277-6214) 11:110-115

Received on 12/5/2020; Accepted on 10/7/2020

doi. 10.33259/JLivestSci.2020.110-115

\begin{abstract}
Pork provides substantial portion of daily meals for human. To meet the global demand for growing population, implementation of advanced technologies in pig breeding is required. The most recently gene editing CRISPR/Cas9 system provides novel tools to pig breeders to improve animal welfare, productivity, and performance. This paper aims to give principles of genome editing techniques and the latest update on CRISPR/Cas9 application on pig industry. We will also review the possible choices for delivering CRISPR/Cas9 system in pig research. It is hoped that with the adaptation of CRISPR/Cas9 technology, rapid genetic understanding and utilization in pig breeding will soon accelerate.
\end{abstract}

Keywords: CRISPR/Cas9; genome editing; animal welfare; pig breeding. 


\section{Introduction}

Providing sufficient food for the constant growing of the human population is currently a challenging mission. In order to feed the projected 9.7 billion people in 2050, the global demand for agricultural products is calculated to increase 60\% 2050 (Alexandratos and Bruinsma 2012). Specifically, a higher animal-based proteins demand for milk and meat outputs is estimated to augment at $60 \%$ and $76 \%$, respectively. Consequently, the livestock industry is required to improve production efficiency in a way that balance the minimizing detrimental influences on the environment and ensuring animal welfares. According to United States Department of Agriculture, domestic pig (Sus scrofa domestica) production provides 42\% of the total livestock production in 2018 (USDA 2018), and $40 \%$ of human consumption of meat sources (Alexandratos and Bruinsma 2012). This clearly reveals a significant contribution of pork in human daily meals. Because of its high finesses in meat quality, pork becomes a regular food in China, United States, and many European and Asian countries.

\section{Traditional breeding and genome engineering in pig breeding}

A myriad of scientific reports has demonstrated various methods to improve pig production to meet the global demand. Classical selective breeding based on measurable traits of economic importance, for instance, feed conversion, litter sizes, carcass fat or meat quality, has tremendously increased the productivity. Specifically, from 1960 s to 2000 s, targeted breeding successfully enhanced $50 \%$ of litter sizes, $37 \%$ of lean pork meat, and doubling the lean pork meat per $\mathrm{kg}$ of feed intake (van der Steen, et al. 2005). Later in the early of $20^{\text {th }}$ century, with the participation of biotechnological solutions, molecular genetic approaches have revolutionized the swine genomic research by the advent of using genetic markers to construct genetic maps for the detection of quantitative trait loci (QTL) and candidate genes of desirable traits. With the aid from increasing powerful computer and available bioinformatics database, hundreds of QTL conferring disease resistance or productivity have been reported in swine during the last twenty years (Elsik, et al. 2009; Groenen, et al. 2012). The introduction of assisted reproductive technologies including artificial insemination (Foote 2002), semen sexing and cryopreservation (Rodríguez-Gil and Estrada 2013; Sathe and Shipley 2014), embryo transfer (Perry 2018), in vitro fertilization (Brackett, et al. 1982) have added more options to breeders to enhance pig production and research. With the rapid development of DNA techniques and animal cloning, genetically modified animals (GMA) has been enabled to emerge (Hammer, et al. 1985; Whitworth, et al. 2016; Burkard, et al. 2018; Whitworth, et al. 2019). These transgenic swine carrying exogenous genes of important agricultural traits have the potential to perform well against diseases and maintain the superior performance. Taken together, classical genetic and biotechnology-based approaches have transformed the swine production and health, and advanced our understanding of mechanisms in swine biology (Schultz, et al. 2020).

However, these methods still have their limitations. While selective breeding and molecular genetic studies are restricted on genetic variation and linkage map within a population (Tait-Burkard, et al. 2018; McFarlane, et al. 2019), assisted reproductive technologies are technically challenging and costly procedures (Hansen 2020). In recombinant DNA and animal cloning methods, many concerns about low efficiency in generating offspring, transgenic animal's health and approval for human consumption have been raised (Yum, et al. 2018; Van Eenennaam, et al. 2019). Most of these obstacles can be met with genome editing approaches. With the emergence of these recently discovered tools, swine breeders are offered a state-of-the-art technology to study and understand genes conferring any traits of interest.

\section{Genome-editing and its principles}

Gene editing is a molecular biology technique that intentionally targets user-defined DNA sites within the genome for the purpose of elucidating functions of unknown genes. Since modified genetic information in the parental lines is passed to next generations, gene editing can be employed to purposely alter traits of agricultural importance to develop new cultivars or breeding lines. Various gene editing techniques have been established including zinc finger nuclease (ZFN), transcription activator-like effector nuclease (TALEN) and cluster regularly interspaced short palindromic repeats (CRISPR)/CRISPR-associated protein 9 (Cas9) (CRISPR/Cas9). All of these tools rely on the specificity of the endonucleases that recognize and cleave DNA at desired sites to facilitate mutations induced by cellular repair mechanism. In this review, we aim to provide the latest updates of CRISPR/Cas9 application on swine breeding, although TALEN and ZFN can obtain the same outcomes.

\section{CRISPR/Cas9 system and its mechanism}

The most recently discovered CRISPR/Cas9 was firstly identified in bacteria as an adaptive defense barrier against foreign DNA. This system has major advantages over the earlier ZFN and TALEN in many ways such as cost-effectiveness, simplicity, greater precision and efficiency (Gupta and Musunuru 2014). It consists of two 
components including CRISPR and Cas9 nuclease complex. In the CRISPR, there are three elements: a leader sequence, an array of repeat sequences and spacer sequences. While the CRISPR locus upstream-localized leader sequence is a specie-specific promoter, the highly conserved palindromic repeats can form hairpin structure (Wu and Bazer 2019). Each repeat in the array is interspaced by a spacer. As the spacer sequences are homologous to the viral sequences or plasmids, they stimulate the cell to capture and degrade the genetic invasion of these corresponding virus of plasmids (Whitworth, et al. 2016). The Cas9 nuclease complex is composed of an endonuclease Cas9 protein, a CRISPR RNA (crRNA) and transactivation CRISPR RNA (tracrRNA). The crRNA is transcribed from the CRISPR locus and subsequently join to tracrRNA to become the single guide RNA (sgRNA). sgRNA and Cas9 protein will form Cas9 complex that recognize and create double-strand exogenous DNA break, thus terminate the invasion (Proudfoot, et al. 2019).

For the gene editing purpose, the programing of sgRNA will determine the genomic location for DNA cleavage, therefore it will facilitate the DNA breakage of modification of chosen genes via DSB. CRISPR/Cas9mediated DSB are recognized by cellular damage repair machinery. During the repairing process, indel mutations are introduced, subsequently inactivates the desired genes of interest via NHEJ pathway. In HR pathway, with the provision of DNA template, homologous recombination will be facilitated, and gene replacement will occur, leading to transgene insertion (Namula, et al. 2019).

\section{Delivery methods of CRISPR/Cas9 system}

To precisely modify the studied genes, accomplishing introduction of CRISPR/Cas9 system into the zygote or reproductive cells is a matter of great importance. Various strategies for CRISPR/Cas9 system transporting can be categorized into two main platforms including viral and non-viral (Huafeng, et al. 2019). In the non-viral platform, physical or chemical approaches, such as electroporation, microinjection, nanoparticles, and hydrodynamic injection, are required to achieve successful CRISPR/Cas9 reagents delivery (Liu, et al. 2017). In the viral platforms, there are three frequently used viral vectors: lentivirus, adenovirus and adeno-associated virus which are utilized as vehicles to incorporate CRISPR/Cas9 system into the cell's genome. Although both platforms possess their own advantages and disadvantages, they have achieved numerous successes in gene-editing research (Liu, et al. 2017). However, according to latest research by McFarlane et al. (2019), of all the methods in two platforms, three latest advanced procedures in reproductive technologies including zygote electroporation, zygote transduction with recombinant adeno-associated virus (rAAV) and surrogate sire technology (SST), will be the most efficient tool in the future that enable efficient transferring CRISPR/Cas9 system into the zygote on farm settings (McFarlane, et al. 2019).

Electroporation is a well-established approach to incorporate foreign reagents into mammalian cells but has recently been improved and optimized for application to zygote in genome editing context. The oocytes from donor super-ovulated females will be collected for in vitro fertilization. After artificial fertilization, zygotes will be subject to electroporation to introduce CRISPR/Cas9 reagents. Subsequently, the electroporated zygotes will be matured in vitro and verified to harbor the genome-edited sequences by screening. Confirmed embryos will be implanted into recipient female to produce genetically modified animals with superior qualities. Due to its computer-based design, online ordering and ready-to-use supplies for CRISPR/Cas9 components, successful genome editing in swine and other animals has been reported (Laible 2018; Miao, et al. 2019; Namula, et al. 2019). Nevertheless, this technique still needs improvement in reduction of mosaicism in the edited offspring, and the length of homologous DNA repair template to induce the HR pathway (McFarlane, et al. 2019).

Because of its non-pathogenicity, high infection efficiency and wide spectrum of cell specificity, employing rAAVs for gene transduction has generated successful results (Kaulich and Dowdy 2015). Following the super ovulated females' oocytes collection and in vitro fertilization, the resulting fertilized zygotes will be immersed in a solution containing rAAVs. The rAAvs will infiltrate the zygote, hijack the cellular mechanism to express the CRISPR/Cas system and induce the genome editing process. Once the rAAVs- edited zygotes are screened by biopsy sequencing, they will be transferred to recipient females to develop into genetically superior animals. Although this approach is still confined in mice research (Yoon, et al. 2018), due to the simplicity for the precision in foreign DNA into the host's genome, rAAV method is considered the most promising method for generating genome-edited livestock in the future (McFarlane, et al. 2019).

The SST involves the production of male animals devoid of endemic germline stem cells. As a result, these animals lack the ability to establish mature sperm. The spermatogonial stem cells (SSCs) will be collected from the donor male and SSCs will undergo CRISPR/Cas9-mediated genetic manipulation to create elite genome edited sperm cells. The cells will be will be transplanted to juvenile sire recipient, resulting a desirable quality sperm bioreactor that can disseminate the traits of interest to progenies by natural breeding (Wang, et al. 2017; Giassetti, et 
al. 2019). In pig industry, SST boars will rapidly disseminate favorably edited genes in a shorter period of time compared to traditional breeding approaches (McFarlane, et al. 2019).

\section{Application of CRISPR/Cas9 system in pig breeding}

Since its introduction, CRISPR/Cas9 system has been widely applied in animal breeding. By 2013, the first pioneering report in genome edition of mammalian cells mediated by CRISPR/Cas9 technique, many animal genomes including cattle, poultry and livestock have been edited. In pig research, the earliest CRISPR/Cas9-based genome-edited pigs were published in 2014. From then on, the expansion of CRISPR/Cas9 system in pig studies continues to offer new opportunities in understanding gene functions and modifying the phenotypes in pig production.

\section{Meat quality}

Myostatin (MSTN) negatively regulates the development of skeletal muscle mass in vivo. In 2015, Wang et al. generated MSTN-knocked out swine by combined utilization of CRISPR/Cas9 system and somatic cell nuclear transfer method (Wang, et al. 2015). In 2016, another report by Bi et al. demonstrated a successful attempt to create MSTN-edited pig by integrating CRISP/Cas9 and Cre/Lox9 approaches (Bi, et al. 2016). Both results from two studies illustrated that genome-edited pigs have higher lean meat production, lower fat accumulation compared to the control pigs. However, there were associating detrimental effects associated with MSTN-null pigs, for instance, motor function impairment in newborn Landrace breed, and piglet's fatality after birth. To circumvent these hurdles, Zou et al. (2018) targeted new candidate gene FBXO40 which influences muscle development. CRISPR/Cas9mediated pigs with nonfunctional FBXO40 gene exhibited similar MSTN-disable phenotypes without previously described deleterious impacts (Zou, et al. 2018). Recently, a study by Xiang et al. (2018) has displayed mutated insulin-like growth factor (IGF2) gene, which stimulates growth and development in mammals during fetal and postnatal stages, resulted in the amelioration of meat quality in indigenous Chinese breed Bama pigs (Xiang, et al. 2018). These results suggested that CRISPR/Cas9 can be applied in pig research to improve meat quality.

\section{Disease resistance}

CRISPR/Cas9 approach has been employed to target CD163, which is the main receptor for porcine reproductive and respiratory syndrome (PRRS) virus. PRRS is one of the most devastating pig diseases which has caused global economic loss during past decades. In 2016, Whitworth et al. reported that CD-163 null pigs displayed complete resistance to both type I and type II PRRS virus isolates (Wells, et al. 2017) and maintained normal biological functions (Whitworth, et al. 2016). Another example in genome editing in generating disease resistance pigs is the directional targeting aminopeptidase $\mathrm{N}$, which is the putative receptor of porcine epidemic diarrhea virus (PEDV) and transmissible gastroenteritis virus (TGEV) (Delmas, et al. 1992; Li, et al. 2007). PEDV and TGEV are causative agents for severe diarrhea in pre-weaned piglets and have been linked to high morbidity and fatality (Proudfoot, et al. 2019). Although edited aminopeptidase N- pigs showed resistance to TGEV, susceptibility to PEDV still remained. Since there is a link between defects of aminopeptidase $\mathrm{N}$ and blood cancers including leukemia and lymphoma, further investigation is needed to scrutinize the effect on health and productivity of the animals (Whitworth, et al. 2019).

\section{Animal welfare}

In pigs, endogenous uncoupling protein 1 (UCP1) participates in thermoregulation during cold stress. Disruption of porcine UCP1 protein will result in the lack of formation of brown adipose tissue and subsequent absence of non-shivering thermogenesis mechanism (Trayhurn, et al. 1989; Berg, et al. 2006; Jastroch and Andersson 2015). Consequently, newborn piglets without functional UCP1 gene are thus prone to cold exposure, which could lead to neonatal fatality. By employment of CRISPR/Cas9-mediated edition UCP1-edited pigs illustrated improvement in cold tolerance (Zheng, et al. 2017).

\section{Conclusion}

Pork is an important source for the increasing demand for better quality and quantity meat protein. As a result, pig industry is pushed to enhance its productivity and reduce environmental impacts. Significant achievements in animal biotechnology have driven the pig breeding programs in many countries with many successes. With the introduction of CRISPR/Cas9 system, pig breeders and researchers are offered a novel tool to rapidly understand traits of great economic significance, augment the pig production and health. It is hoped that CRISPR/Cas9 system will accelerate the research progress in pig industry in the next coming decades. 
Tinh et al 2020/ J. Livestock Sci. 11: 110-115

Acknowledgement -The authors are in debt to Institute of Animal Sciences for Southern Vietnam for their support during manuscript preparation.

Conflict of interest declaration - None

\section{References}

1) Alexandratos N, Bruinsma J. 2012. World Agriculture Towards 2030/2050: The 2012 Revision. ESA Working paper No. 12-03. Rome: FAO.

2) Berg F, Gustafson U, Andersson L. 2006. The Uncoupling Protein 1 Gene (UCP1) Is Disrupted in the Pig Lineage: A Genetic Explanation for Poor Thermoregulation in Piglets. PLOS Genetics 2:e129.

3) Bi Y, Hua Z, Liu X, Hua W, Ren H, Xiao H, Zhang L, Li L, Wang Z, Laible G, Wang Y, Dong F, Zheng X. 2016. Isozygous and selectable marker-free MSTN knockout cloned pigs generated by the combined use of CRISPR/Cas9 and Cre/LoxP. Scientific reports 6:31729-31729.

4) Brackett BG, Bousquet D, Boice ML, Donawick WJ, Evans JF, Dressel MA. 1982. Normal Development Following In Vitro Fertilization in the Cow. Biology of Reproduction 27:147-158.

5) Burkard C, Opriessnig T, Mileham AJ, Stadejek T, Ait-Ali T, Lillico SG, Whitelaw CBA, Archibald AL. 2018. Pigs Lacking the Scavenger Receptor Cysteine-Rich Domain 5 of CD163 Are Resistant to Porcine Reproductive and Respiratory Syndrome Virus 1 Infection. Journal of Virology 92:e00415-00418.

6) Delmas B, Gelfi J, L'Haridon R, Vogel, Sjöström H, Norén, Laude H. 1992. Aminopeptidase N is a major receptor for the enteropathogenic coronavirus TGEV. Nature 357:417-420.

7) Elsik CG, Tellam RL, Worley KC. 2009. The Genome Sequence of Taurine Cattle: A Window to Ruminant Biology and Evolution. Science 324:522-528.

8) Foote RH. 2002. The history of artificial insemination: Selected notes and notables1. Journal of Animal Science 80:1-10.

9) Giassetti MI, Ciccarelli M, Oatley JM. 2019. Spermatogonial Stem Cell Transplantation: Insights and Outlook for Domestic Animals. Annual Review of Animal Biosciences 7:385-401.

10) Groenen MAM, Archibald AL, Uenishi H, Tuggle CK, Takeuchi Y, Rothschild MF, Rogel-Gaillard C, Park C, Milan D, et al 2012. Analyses of pig genomes provide insight into porcine demography and evolution. Nature 491:393-398.

11) Gupta RM, Musunuru K. 2014. Expanding the genetic editing tool kit: ZFNs, TALENs, and CRISPR-Cas9. The Journal of clinical investigation 124:4154-4161.

12) Hammer RE, Pursel VG, Rexroad CE, Wall RJ, Bolt DJ, Ebert KM, Palmiter RD, Brinster RL. 1985. Production of transgenic rabbits, sheep and pigs by microinjection. Nature 315:680-683.

13) Hansen PJ. 2020. Implications of Assisted Reproductive Technologies for Pregnancy Outcomes in Mammals. Annual Review of Animal Biosciences 8:395-413.

14) Huafeng L, Qiudi D, Lili L, Lei S. 2019. Application and Development of CRISPR/Cas9 Technology in Pig Research. IntechOpen.

15) Jastroch M, Andersson L. 2015. When pigs fly, UCP1 makes heat. Molecular Metabolism 4:359-362.

16) Kaulich M, Dowdy SF. 2015. Combining CRISPR/Cas9 and rAAV Templates for Efficient Gene Editing. Nucleic Acid Therapeutics 25:287-296.

17) Laible G. 2018. Production of Transgenic Livestock: Overview of Transgenic Technologies. In: Niemann H, Wrenzycki C, editors. Animal Biotechnology 2: Emerging Breeding Technologies. Cham: Springer International Publishing. p. 95-121.

18) Li BX, Ge JW, Li YJ. 2007. Porcine aminopeptidase N is a functional receptor for the PEDV coronavirus. Virology 365:166-172.

19) Liu C, Zhang L, Liu H, Cheng K. 2017. Delivery strategies of the CRISPR-Cas9 gene-editing system for therapeutic applications. Journal of controlled release : official journal of the Controlled Release Society 266:17-26.

20) McFarlane GR, Salvesen HA, Sternberg A, Lillico SG. 2019. On-Farm Livestock Genome Editing Using Cutting Edge Reproductive Technologies. Frontiers in Sustainable Food Systems 3.

21) Miao D, Giassetti MI, Ciccarelli M, Lopez-Biladeau B, Oatley JM. 2019. Simplified pipelines for genetic engineering of mammalian embryos by CRISPR-Cas9 electroporation†. Biology of Reproduction 101:177187.

22) Namula Z, Wittayarat M, Hirata M, Hirano T, Nguyen NT, Le QA, Fahrudin M, Tanihara F, Otoi T. 2019. Genome mutation after the introduction of the gene editing by electroporation of Cas 9 protein (GEEP) system into bovine putative zygotes. In Vitro Cellular \& Developmental Biology - Animal 55:598-603. 
23) Perry G. 2018. 2016 statistics of embryo collection and transfer in domestic farm animals. In: The International Embryo Transfer Society Data Retrieval Committee.

24) Proudfoot C, Lillico S, Tait-Burkard C. 2019. Genome editing for disease resistance in pigs and chickens. Animal Frontiers 9:6-12.

25) Rodríguez-Gil J, Estrada E. 2013. Artificial insemination in boar reproduction. In: Boar Reproduction:589-607.

26) Sathe S, Shipley CF. 2014. Cryopreservation of Semen. In. Bovine Reproduction. p. 662-670.

27) Schultz B, Serão N, Ross JW. 2020. Chapter 23 - Genetic improvement of livestock, from conventional breeding to biotechnological approaches. In: Bazer FW, Lamb GC, Wu G, editors. Animal Agriculture: Academic Press. p. 393-405.

28) Tait-Burkard C, Doeschl-Wilson A, McGrew MJ, Archibald AL, Sang HM, Houston RD, Whitelaw CB, Watson M. 2018. Livestock 2.0 - genome editing for fitter, healthier, and more productive farmed animals. Genome Biology 19:204.

29) Trayhurn P, Temple NJ, Aerde JV. 1989. Evidence from immunoblotting studies on uncoupling protein that brown adipose tissue is not present in the domestic pig. Canadian Journal of Physiology and Pharmacology 67:1480-1485.

30) USDA. 2018. Livestock and Poultry. World Markets and Trade: U.S. Department of Agriculture [Internet].

31) van der Steen HAM, Prall GFW, Plastow GS. 2005. Application of genomics to the pork industry. Journal of Animal Science 83:E1-E8.

32) Van Eenennaam AL, Wells KD, Murray JD. 2019. Proposed U.S. regulation of gene-edited food animals is not fit for purpose. npj Science of Food 3:3.

33) Wang K, Ouyang H, Xie Z, Yao C, Guo N, Li M, Jiao H, Pang D. 2015. Efficient Generation of Myostatin Mutations in Pigs Using the CRISPR/Cas9 System. Scientific reports 5:16623-16623.

34) Wang Y, Ding Y, Li J. 2017. CRISPR-Cas9-Mediated Gene Editing in Mouse Spermatogonial Stem Cells. In: Zhang B, editor. RNAi and Small Regulatory RNAs in Stem Cells: Methods and Protocols. New York, NY: Springer New York. p. 293-305.

35) Wells KD, Bardot R, Whitworth KM, Trible BR, Fang Y, Mileham A, Kerrigan MA, Samuel MS, Prather RS, Rowland RRR. 2017. Replacement of Porcine CD163 Scavenger Receptor Cysteine-Rich Domain 5 with a CD163-Like Homolog Confers Resistance of Pigs to Genotype 1 but Not Genotype 2 Porcine Reproductive and Respiratory Syndrome Virus. Journal of Virology 91:e01521-01516.

36) Whitworth KM, Rowland RRR, Ewen CL, Trible BR, Kerrigan MA, Cino-Ozuna AG, Samuel MS, Lightner JE, McLaren DG, Mileham AJ, Wells KD, Prather RS. 2016. Gene-edited pigs are protected from porcine reproductive and respiratory syndrome virus. Nature Biotechnology 34:20-22.

37) Whitworth KM, Rowland RRR, Petrovan V, Sheahan M, Cino-Ozuna AG, Fang Y, Hesse R, Mileham A, Samuel MS, Wells KD, Prather RS. 2019. Resistance to coronavirus infection in amino peptidase N-deficient pigs. Transgenic Research 28:21-32.

38) Wu G, Bazer FW. 2019. Application of new biotechnologies for improvements in swine nutrition and pork production. Journal of animal science and biotechnology 10:28.

39) Xiang G, Ren J, Hai T, Fu R, Yu D, Wang J, Li W, Wang H, Zhou Q. 2018. Editing porcine IGF2 regulatory element improved meat production in Chinese Bama pigs. Cellular and Molecular Life Sciences 75:46194628.

40) Yoon Y, Wang D, Tai PWL, Riley J, Gao G, Rivera-Pérez JA. 2018. Streamlined ex vivo and in vivo genome editing in mouse embryos using recombinant adeno-associated viruses. Nature Communications 9:412.

41) Yum S-Y, Youn K-Y, Choi W-J, Jang G. 2018. Development of genome engineering technologies in cattle: from random to specific. Journal of animal science and biotechnology 9:16-16.

42) Zheng Q, Lin J, Huang J, Zhang H, Zhang R, Zhang X, Cao C, Hambly C, Qin G, Yao J, Song R, Jia Q, Wang X, Li Y, Zhang N, Piao Z, Ye R, Speakman JR, Wang H, Zhou Q, Wang Y, Jin W, Zhao J. 2017. Reconstitution of UCP1 using CRISPR/Cas9 in the white adipose tissue of pigs decreases fat deposition and improves thermogenic capacity. Proceedings of the National Academy of Sciences of the United States of America 114:E9474-E9482.

43) Zou Y, Li Z, Zou Y, Hao H, Li N, Li Q. 2018. An FBXO40 knockout generated by CRISPR/Cas9 causes muscle hypertrophy in pigs without detectable pathological effects. Biochemical and Biophysical Research Communications 498:940-945. 